\title{
Temperature changes of the exciton transition energy in lead di-iodide nanofilms
}

\author{
O.V. Pugantseva ${ }^{1}$, V.M. Kramar ${ }^{1}$, I.V. Fesiv ${ }^{1}$, O.O. Kudryavtsev ${ }^{2}$ \\ ${ }^{1} Y u$. Fed'kovych Chernivtsi National University, \\ 2, vul. Kotsiubynskogo, 58012 Chernivtsi, Ukraine; \\ e-mail:v.kramar@chnu.edu.ua \\ ${ }^{2} V$. Lashkaryov Institute of Semiconductor Physics, NAS of Ukraune, \\ 41, prospect Nauky, 03028 Kyiv, Ukraine; \\ e-mail: journal@isp.kiev.ua
}

\begin{abstract}
Adduced in this work are the results of theoretical investigations devoted to the influence of spatial confinement effects, self-polarization of heterojunction planes and exciton-phonon interaction on values of the exciton transition energy in lead diiodide nanofilms placed into dielectric ambience (glass or polymer). The heterojunction is considered to be unloaded, nanosystem is modeled with an infinite deep quantum well and characterized by an essential difference between dielectric permeabilities in both sides of the heterojunction. Calculated in the work are dependences of the exciton energy on the nanofilm thickness and temperature. The results of calculations are in accordance with the known data of experimental measurements.
\end{abstract}

Keywords: nano-heterostructure, quantum well, nanofilm, exciton, transition energy, layered semiconductor, lead di-iodide.

Manuscript received 16.01.13; revised version received 20.02.13; accepted for publication 19.03.13; published online 25.06.13.

\section{Introduction}

Lead di-iodide is promising material for creation detectors of $\mathrm{X}$ - and $\gamma$-radiations, which are capable to operate in the wide $\left(-200\right.$ up to $\left.+130{ }^{\circ} \mathrm{C}\right)$ temperature interval $[1,2]$. It explains hasty development of technologies for preparation and investigations of physical properties both of bulk lead di-iodide crystals $[3,4]$ and low-dimensional [5], in particular, hybrid nonorganic - organic heterostructures based on it [6-10]. The latter are especially interesting due to combination of properties inherent to materials of both types as well as availability of spatial confinement (SC) effects.

Spatial confinement of quasi-particles in lowdimensional structures causes additional quantization of their motion characteristics, which can be observed in changes of their optical properties, including those in the exciton range of spectrum $[5-7,11]$.

Theoretical investigations of exciton states in nanostructures with single quantum wells (QW) were performed by many authors that used various methods and approaches (see, for instance, [12-15]), however, the nanostructures based on lead di-iodide have not been considered there.

This paper represents the results of theoretical investigations of temperature changes in the energy of transition into the ground exciton state observed in a plane nano-film (NF) made of 2H-polytype of lead diiodide $\left(2 \mathrm{H}-\mathrm{PbI}_{2}\right)$ embedded into dielectric medium. As the latter, we considered borosilicate glass and copolymer of ethylene-metacryle acid (E-MAA). Lowdimensional crystal structures of this type were prepared and investigated in the works [5-7]. In particular, experimentally measured in [5] are the positions of exciton minimums in transmission spectra of microcrystals grown in voids of the matrix consisting of borosilicate glass, while observed in [6] were exciton peaks in low-temperature $(T=2 \mathrm{~K})$ absorption spectra of $\mathrm{PbI}_{2}$ microcrystals shaped as hexagonal plates synthesized using the thermo-chemical method in E- 
MAA medium. In both experiments, there were investigated microcrystals with the thickness up to 7 layers of lead di-iodide and found was the dependence of exciton peak positions on the thickness - when the latter is increased the exciton peak (which is short-wave shifted relatively to its position in the bulk crystal) shifts to the side of long waves. In accord with [5], there were no essential changes in peak positions when heating the sample up to $77 \mathrm{~K}$, while the further increase up to the room temperature resulted in their shift to the long-wave side by $80 \mathrm{meV}$.

Taking into account that the microcrystal thickness is considerably smaller than its transverse sizes, one can consider this structure as quasi-two-dimensional. Modeling it with a plane double heterostructure of the first type with infinite deep QWs - both the electron and hole ones - that is by a nanofilm, we obtained analytical expressions that allowed calculating the energy of transition into the ground exciton state in NF with various thicknesses at different temperatures.

It has been shown that increasing the NF thickness causes a non-linear shift of the exciton transition energy into the range of low values, and the higher is the temperature, the more essential this shift, which is in accordance with the data of measurements $[5,6]$.

\section{Considering the model of investigation and setting the task}

We consider the model of the Wannier-Mott exciton in a plane semiconductor nano-heterostructure with a double heterojunction - NP (medium 0) embedded into isotropic dielectric (medium 1). Layered semiconductors possess atomically-flat surface with a small number of broken bonds [16], therefore, the heterojunction will be considered as unloaded, and QW as rectangular. With account of dielectric nature of barrier medium, QW should be considered as infinitly deep.

We chose the system of Cartesian coordinates in such a manner that its origin was placed in the QW center, XOY plane was parallel to its surface, and $\mathrm{OZ}$ axis coincided with the crystallographic axis $\mathrm{C}$ of the layered crystal. If $c$ is the lattice constant along the direction of $C$-axis, then the QW thickness (equal to the QW width) is $N c$, where $N$ is a natural number equal to the number of layers in $\mathrm{QW}$ material.

As usual, theoretical investigations of semiconductor nano-heterostructures are performed in the approach of effective masses for the electron subsystem and within the model of dielectric continuum - for the phonon one [17-19], while the Hamiltonian for the exciton-phonon system has the form

$\hat{H}=\hat{H}_{e x}+\hat{H}_{p h}+\hat{H}_{\text {int }}$,

where $\hat{H}_{e x}$ and $\hat{H}_{p h}$ operators describe, respectively, states of the free exciton and phonon, and $\hat{H}_{\text {int }}-$ their interaction.
In the coordinate system related with the exciton mass center its Hamiltonian takes the following form

$\hat{H}_{\mathrm{ex}}=-\frac{\hbar^{2} \nabla_{\rho}^{2}}{2 \mu}+\sum_{p=e, h} \hat{H}_{p}\left(z_{p}\right)+V_{\mathrm{e}-\mathrm{h}}\left(\vec{\rho}, z_{e}, z_{h}\right)$,

where

$\hat{H}_{p}\left(z_{p}\right)=-\frac{\hbar^{2}}{2 m_{p \|}} \frac{\partial^{2}}{\partial z_{p}^{2}}+V\left(z_{p}\right)+V_{\mathrm{sp}}\left(z_{p}\right)$

is the single-particle Hamiltonian that describes motion of an electron $(p=e)$ or hole $(p=h)$ in the respective QW, which is set by the limiting potential

$V\left(z_{p}\right)= \begin{cases}0, & \left|z_{p}\right|<N c / 2 \\ \infty, & \left|z_{p}\right| \geq N c / 2 .\end{cases}$

Here,

$V_{\mathrm{sp}}\left(z_{p}\right)=\frac{e^{2}}{2 \varepsilon_{\infty}^{(0)}} \sum_{n \neq 0} \frac{\xi^{|n|}}{\left|z_{p}-(-1)^{n} z_{p}+n a\right|}$

is the potential describing interaction of a carrier with its electrostatic image in planes of heterojunctions (selfpolarization (SP) potential $[20,21])$;

$$
\begin{aligned}
& V_{\mathrm{e}-\mathrm{h}}\left(\vec{\rho}, z_{e}, z_{h}\right)=-\frac{e^{2}}{\varepsilon_{\infty \perp}^{(0)} \sqrt{\rho^{2}+\left[\varepsilon_{\infty\|\|}^{(0)}\left(z_{e}-z_{h}\right) / \varepsilon_{\infty \perp}^{(0)}\right]^{2}}}- \\
& -\frac{e^{2}}{\varepsilon_{\infty \perp}^{(0)}} \sum_{n \neq 0} \frac{\xi^{|n|}}{\sqrt{\rho^{2}+\left\{\varepsilon_{\infty}^{(0)}\left[z_{e}-(-1)^{n} z_{h}+n N c\right] / \varepsilon_{\infty \perp}^{(0)}\right\}^{2}}}
\end{aligned}
$$

is the potential of Coulomb interaction between an electron and hole as well as with electrostatic images of both quasi-particles in the planes of heterojunctions. This potential is adapted for the case of layered semiconductor; $\quad m_{p \|}\left(m_{p_{\perp}}\right) \quad$ and $\varepsilon_{\infty \|}^{(l)}\left(\varepsilon_{\infty \perp}^{(l)}\right)$ are, respectively, the effective mass of a carrier and dynamic dielectric permeability of specific medium (inside QW for $l=0$, or the barrier one for $l=1$ ) that are characteristic for the directions along (transverse) the crystallographic $C$-axis; vector $\vec{\rho}=\left(x_{e}-x_{h}, y_{e}-y_{h}\right)$ defines the mutual arrangement of the electron and hole in QW within the XOY plane

$\xi=\frac{\varepsilon_{\infty\|\|}^{(0)}-\varepsilon_{\infty \|}^{(1)}}{\varepsilon_{\infty \|}^{(0)}+\varepsilon_{\infty \|}^{(1)}}$.

To find an exact solution for the Schroedinger equation with the Hamiltonian (1) is impossible, since its intrinsic functions are unknown. Therefore, the energy of exciton transition (without account of its interaction with phonons) can be represented in the following sum: $E_{\mathrm{ex}}^{(0)}=E_{g}+E^{(\mathrm{e})}+E^{(\mathrm{h})}-E_{b}$,

where $E_{g}$ is the width of the forbidden band in QW material; $E^{(\mathrm{e})}$ and $E^{(\mathrm{h})}$ are energies of an electron and hole in $\mathrm{QW}$, respectively; $E_{b}$ is the binding energy, the 
approximate value of which can be found using one of variational methods applied, for instance, in [12-15].

The Hamiltonians for free phonons and excitonphonon interaction (EPI) (in coordinate representation by electron variables) have been obtained in the works $[17,18]$. For the system under consideration, the former takes the following look:

$$
\begin{aligned}
& \hat{H}_{\mathrm{ph}}=\hat{H}_{\mathrm{LO}}+\hat{H}_{\mathrm{I}}=\sum_{\lambda, \vec{q}_{\perp}} \Omega_{\mathrm{LO}}\left(\hat{b}_{\lambda \vec{q}_{\perp}}^{+} \hat{b}_{\lambda \vec{q}_{\perp}}+1 / 2\right)+ \\
& +\sum_{\sigma=S, A} \sum_{\vec{q}_{\perp}} \Omega_{\sigma}\left(\vec{q}_{\perp}\right)\left(\hat{b}_{\sigma \vec{q}_{\perp}}^{+} \hat{b}_{\sigma \vec{q}_{\perp}}+1 / 2\right)
\end{aligned}
$$

where $\Omega_{\mathrm{LO}}$ is the energy of longitudinal optical polarization (LO) phonons limited in QW medium; $\hat{b}_{\lambda \vec{q} \perp}^{+}$and $\hat{b}_{\lambda \vec{q}_{\perp}}$ are operators of birth and annihilation for the LO-phonon state with the wave-vector $\vec{q}=\left(q_{\|}, \vec{q}_{\perp}\right)$, which longitudinal component $\left(q_{\|}\right)$is quantized acquiring discrete values from the set $\lambda \pi / N_{c}(\lambda=1,2, \ldots, N)$;

$\Omega_{\sigma}\left(\vec{q}_{\perp}\right)=\sqrt{\frac{\epsilon_{\sigma}^{(0)}\left(\vec{q}_{\perp}\right) \Omega_{\mathrm{LO}}^{2}+\epsilon_{\sigma}^{(1)}\left(\vec{q}_{\perp}\right) \Omega_{\mathrm{TO}}^{2}}{\epsilon_{\sigma}^{(0)}\left(\vec{q}_{\perp}\right)+\epsilon_{\sigma}^{(1)}\left(\vec{q}_{\perp}\right)}}$

are energies of interface (I) phonons for symmetric $(\sigma=\mathrm{S})$ or antisymmetric $(\sigma=\mathrm{A})$ modes.

Here,

$\epsilon^{(0)}\left\{=\left\{\begin{array}{l}S \\ A\end{array}\right\}\left(\vec{q}_{\perp}\right)=\varepsilon_{\infty \perp}^{(0)}\left[1 \mp \exp \left(-N c q_{\perp}\right)\right]\right.$,
$\epsilon^{(1)}$
$\sigma=\left\{\begin{array}{l}S \\ A\end{array}\right\}$

$\Omega_{\mathrm{TO}}=\Omega_{\mathrm{LO}} \sqrt{\varepsilon_{\infty} / \varepsilon_{0}}$ is the energy of transversal optical phonons, and $\hat{b}_{\sigma \vec{q}_{\perp}}\left(\hat{b}_{\sigma \vec{q}_{\perp}}\right)$ are operators of birth (annihilation) for the respective I-phonon states.

The Hamiltonian for exciton-phonon interaction (EPI) $[17,18]$ used by the authors of [22] before representation within the framework of secondary quantization by all the variables of the electron-phonon system

$\hat{H}_{\text {int }}=\hat{H}_{\mathrm{e}-\mathrm{LO}}+\hat{H}_{\mathrm{e}-\mathrm{I}}=$

$=\sum_{p=e, h} \eta_{p}\left\{\sum_{n_{p}, n_{p}^{\prime}, \vec{k}_{\perp}}\left[\sum_{\lambda, \vec{q}_{\perp}} F_{n_{p} n_{p}^{\prime}}^{\lambda}\left(\vec{q}_{\perp}\right) \hat{a}_{n_{p}^{\prime} \vec{k}_{\perp}+\vec{q}_{\perp}} \hat{a}_{n_{p} \vec{k}_{\perp}} \hat{B}_{\lambda \vec{q}_{\perp}}+\right.\right.$

$\left.\left.+\sum_{\sigma, \vec{q}_{\perp}} F_{n_{p} n_{p}^{\prime}}^{\sigma}\left(\vec{q}_{\perp}\right) \hat{a}_{n_{p}^{\prime} \vec{k}_{\perp}+\vec{q}_{\perp}} \hat{a}_{n_{p} \vec{k}_{\perp}} \hat{B}_{\sigma \vec{q}_{\perp}}\right]\right\}$

is defined by the functions of electron-phonon bond $F_{n n^{\prime}}^{\alpha}\left(\vec{q}_{\perp}\right)$ which explicit look for a QW with a finite depth is shown in [22], and with the infinite one - in [23]. Here, $\eta_{e}=1, \eta_{h}=-1 ; n$ and $n^{\prime}$ are the numbers of subbands for an electron in the state $\left(n, \vec{k}_{\perp}\right) ; \alpha$ is an index of the phonon branch, which is equal to $\lambda$ of LO-phonons or $\sigma-$ in the case of I-phonons; $\hat{B}_{\alpha \vec{q}_{\perp}}=\hat{b}_{\alpha \vec{q}_{\perp}}+\hat{b}_{\alpha-\vec{q}_{\perp}}^{+}$is phonon operator of secondary quantization, while $\hat{a}_{n \vec{k}_{\perp}}$ and $\hat{a}_{n \vec{k}_{\perp}}^{+}$are electron operators of secondary quantization.

The Hamiltonian (3) is obtained in the assumption that EPI is realized via individual interaction of the electron and hole, which forms the exciton, with phonons. This interaction re-normalizes the energy spectrum of quasi-particles, which causes a shift of their states along the energy scale. The value of this shift for the bottom of the carrier $n$-th subband $E_{n}=E_{n}\left(\vec{k}_{\perp}=0\right)$ is defined by the position of poles inherent to the Fourier-transform for the respective Green function

$$
G_{n}\left(\vec{k}_{\perp}, E, T\right)=\frac{1}{E-E_{n}\left(\vec{k}_{\perp}\right)-M_{n}\left(\vec{k}_{\perp}, E, T\right)},
$$

where $M_{n}\left(\vec{k}_{\perp}, E, T\right) \equiv M_{n n}\left(\vec{k}_{\perp}, E, T\right)$ are the diagonal matrix elements for the mass operator (MO) of the electron-phonon system, in which electron-phonon interaction is taken into account for arbitrary temperatures [19]. Determining it for a specific model of nanosystem allows to determine the shift value for the bottom of the $n$ th sub-band of an electron $(p=e)$ or hole $(p=h)$ in QW

$$
\Delta_{n}^{(p)}(T) \equiv-M_{n}^{(p)}\left(\vec{k}_{\perp}=0, E=E_{n}^{(p)}, T\right) .
$$

The energy of transition into the ground exciton state is also shifted into the long-wave range by the value equal to the sum of shifts $\Delta^{(e, h)}(T)$ in the energies $E_{1}^{(e, h)}$ of ground states for electrons and holes, which values depend on temperature

$E_{e x}=E_{g}+E_{1}^{(e)}+E_{1}^{(h)}-E_{b}-\Delta^{(e)}(T)-\Delta^{(h)}(T)$.

The aim of this work is to study temperature changes in the energy $E_{e x}$ for NF prepared from layer semiconductor $\mathrm{PbI}_{2}$ embedded into isotropic dielectric medium with account of the SC, SP and EPI effects.

\section{Calculation method and results obtained}

To perform the set task, we shall limit ourselves with a single-phonon approximation in the model of electrophonon system $[19,24]$. Being adapted for the model under studying, it has a look

$$
\begin{aligned}
& M\left(\vec{k}_{\perp}, E, T\right)=\left.\sum_{\alpha, n, \vec{q}_{\perp}} F_{1 n}^{\alpha}\left(\vec{q}_{\perp}\right)\right|^{2} \times \\
& \times\left[\frac{1+v_{\alpha}\left(\vec{q}_{\perp}, T\right)}{E-E_{n}\left(\vec{k}_{\perp}-\vec{q}_{\perp}\right)-\Omega_{\alpha}\left(\vec{q}_{\perp}\right)}+\frac{v_{\alpha}\left(\vec{q}_{\perp}, T\right)}{E-E_{n}\left(\vec{k}_{\perp}-\vec{q}_{\perp}\right)+\Omega_{\alpha}\left(\vec{q}_{\perp}\right)}\right],
\end{aligned}
$$


where $\alpha$ is the index of a phonon branch, $v_{\alpha}\left(\vec{q}_{\perp}, T\right)=\left\{\exp \left[\Omega_{\alpha}\left(\vec{q}_{\perp}\right) / k_{\mathrm{B}} T\right]-1\right\}^{-1}$ are the numbers of filling the respective phonon states at a temperature $T$, and $k_{\mathrm{B}}$ is the Boltsmann constant.

Using the explicit look of the functions describing electron-phonon binding and transferring from the sum by transverse components of the phonon wavevector $\vec{q}_{\perp}$ to the integral, one can deduce (using (5)) the expressions for determining the temperature dependence of the shift value for the bottom of the electron (hole) band due to interaction between both types of phonons. The value of the shift caused by interaction of a carrier from the ground mini-band with LO-phonons is defined by the following expression

$$
\begin{aligned}
& \Delta_{\mathrm{LO}}(T)=\frac{4 e^{2}}{\pi^{2} \varepsilon_{0}^{(0)} N c} \sum_{n} \sum_{\lambda=1}^{N} \lambda^{2}\left|X_{1 n}^{\lambda}\right|^{2} \times \\
& \times\left\{\left[1+v_{\mathrm{LO}}(T)\right] \Phi_{\lambda n}^{(+)}\left(E_{1}\right)+v_{\mathrm{LO}}(T) \Phi_{\lambda n}^{(-)}\left(E_{1}\right)\right\},
\end{aligned}
$$

where

$$
X_{1 n}^{\lambda}=\left\{\begin{array}{lr}
\frac{\cos \frac{1-n}{2} \pi}{\lambda^{2}-(1-n)^{2}}+\frac{\cos \frac{1+n}{2} \pi}{\lambda^{2}-(1+n)^{2}}(\lambda=1,3, \ldots)- \\
\text { for odd } n ; \\
\frac{\sin \frac{1-n}{2} \pi}{\lambda^{2}-(1-n)^{2}}-\frac{\sin \frac{1+n}{2} \pi}{\lambda^{2}-(1+n)^{2}} & (\lambda=2,4, \ldots)- \\
& \text { for even } n ;
\end{array}\right.
$$$$
\Phi_{\lambda n}^{( \pm)}(E)=\frac{\ln \frac{\left[1+(N c / \lambda a)^{2}\right]\left|1 \pm \zeta_{n}(E)\right|}{\eta+\zeta_{n}(E)+1}}{(\lambda a / N c)^{2} \eta-\zeta_{n}(E) \mp 1} ;
$$$$
\eta=\frac{\pi^{2} \hbar^{2}}{2 m_{\perp} a^{2} \Omega_{\mathrm{LO}}} ; \zeta_{n}(E)=\frac{E_{n}-E}{\Omega_{\mathrm{LO}}} ;
$$

$a$ and $m_{\perp}$ are, respectively, the lattice parameter and carrier effective mass along the direction perpendicular to the $C$-axis. By analogy, for the case of interaction between this carrier with I-phonons

$$
\begin{aligned}
& \Delta_{\mathrm{I}}(T)=\frac{2 e^{2}}{N c} \int_{0}^{\pi / a}\left|f_{1 n}^{\sigma}\left(\vec{q}_{\perp}\right)\right|^{2} \Phi_{\sigma}\left(\vec{q}_{\perp}\right) \times \\
& \times\left[\frac{1+\mathrm{v}_{v}\left(\vec{q}_{\perp}, T\right)}{\eta_{\sigma}\left(\vec{q}_{\perp}\right)+\zeta_{n \sigma}\left(\vec{q}_{\perp}\right)+1}+\frac{\mathrm{v}_{v}\left(\vec{q}_{\perp}, T\right)}{\eta_{\sigma}\left(\vec{q}_{\perp}\right)+\zeta_{n \sigma}\left(\vec{q}_{\perp}\right)-1}\right] d q_{\perp},
\end{aligned}
$$

where

$$
\begin{aligned}
& f_{1 n}^{\sigma}\left(\vec{q}_{\perp}\right)=\sqrt{1+\exp \left(-N c q_{\perp}\right)} \cdot \delta_{\sigma S} \cdot N c q_{\perp} \tanh \left(\frac{N c q_{\perp}}{2}\right) \times \\
& \times\left\{\frac{\cos \frac{(1-n) \pi}{2}}{[(1-n) \pi]^{2}+\left[N c q_{\perp}\right]^{2}}+\frac{\cos \frac{(1+n) \pi}{2}}{[(1+n) \pi]^{2}+\left[N c q_{\perp}\right]^{2}}\right\} .
\end{aligned}
$$

For odd $n$ and

$$
\begin{aligned}
& f_{1 n}^{\sigma}\left(\vec{q}_{\perp}\right)=-\sqrt{1-\exp \left(-N c q_{\perp}\right)} \cdot \delta_{\sigma A} \cdot N c q_{\perp} \operatorname{coth} \frac{q_{\perp} a}{2} \times \\
& \times\left\{\frac{\sin \frac{(1-n) \pi}{2}}{[(1-n) \pi]^{2}+\left[N c q_{\perp}\right]^{2}}+\frac{\sin \frac{(1+n) \pi}{2}}{[(1+n) \pi]^{2}+\left[N c q_{\perp}\right]^{2}}\right\}
\end{aligned}
$$

- for the even ones;

$$
\begin{aligned}
& \Phi_{\sigma}\left(\vec{q}_{\perp}\right)=\frac{\left[\Omega_{\mathrm{TO}}^{2}-\Omega_{\sigma}^{2}\left(\vec{q}_{\perp}\right)\right]^{2}}{\varepsilon_{\infty \perp}^{(0)} \Omega_{\sigma}^{2}\left(\vec{q}_{\perp}\right)\left(\Omega_{\mathrm{LO}}^{2}-\Omega_{\mathrm{TO}}^{2}\right)} ; \\
& \eta_{\sigma}\left(\vec{q}_{\perp}\right)=\frac{\hbar^{2} q_{\perp}^{2}}{2 m_{\perp} \Omega_{\sigma}\left(\vec{q}_{\perp}\right)} ; \varsigma_{n \sigma}\left(\vec{q}_{\perp}\right)=\frac{E_{n}-E}{\Omega_{\sigma}\left(\vec{q}_{\perp}\right)} .
\end{aligned}
$$

The value of the total shift inherent to the bottom of the carrier ground mini-band in its $\mathrm{QW}$ is equal to the sum of partial shifts (7) and (8); the shift of the bottom for the ground exciton band can be expressed, respectively,

$$
\Delta(T)=\Delta_{\mathrm{LO}}(T)+\Delta_{\mathrm{I}}(T)=\sum_{p=e, h}\left[\Delta_{\mathrm{LO}}^{(p)}(T)+\Delta_{\mathrm{I}}^{(p)}(T)\right] .
$$

In this work, specific calculations were performed for $2 \mathrm{H}-\mathrm{PbI}_{2} \mathrm{NF}$ (lattice parameters $a=4.557 \AA$, $c=6.979 \AA$; forbidden gap $E_{g}=2.5 \mathrm{eV}$; energy of LOphonons $\Omega_{\mathrm{LO}}=109 \mathrm{~cm}^{-1}$ [25]; dielectric permeabilities $\varepsilon_{0 \|}=9.3 ; \varepsilon_{0 \perp}=26.4 ; \varepsilon_{\infty\|\|}=6.0 ; \varepsilon_{\infty \perp}=6.25$ [26]; effective masses of electrons and holes determined for the directions along C-axis $\left(m_{e \|}=1.25\right.$ and $m_{h \|}=1.25$ [27]) as well as transverse to it $\left(m_{e_{\perp}}=0.28\right.$ and $m_{h_{\perp}}=0.40$ [28])) embedded into E-MAA polymer or glass. Dielectric permeabilities of these substances are considered as independent of the frequency and equal 2.38 for E-MAA [29] and 4.7 for borosilicate glass. The results of calculations are adduced in Figs $1-3$.

The absolute value of the long-wave shift of the bottom for the ground exciton mini-band, which is caused by interaction with LO-phonons, was calculated for NF of various thicknesses and low temperatures (in the vicinity of absolute zero) and shown in Fig. 1 (curves 1 ). Since $\Delta_{\text {LO }}$ is exclusively determined by parameters of the well (lead di-iodide), for both nanostructures $\mathrm{PbI}_{2} /$ E-MAA (Fig. 1, to the left) or $\mathrm{PbI}_{2} /$ glass (Fig. 1, to the right) these shifts are identical.

The values of shifts caused by interaction with Iphonons are different - in the first nanostructure $\Delta_{I}$ values are essentially higher than in the second one (curves 2). It is related with the fact that the functions of relations with the interface phonons are defined by parameters not only of well material but barrier one, as well. Therefore, the difference in dielectric permeabilities of E-MAA medium and glass is pronounced in differences of respective $\Delta_{\mathrm{I}}$ values. It is clear that the total shift $\Delta=\Delta_{\mathrm{LO}}+\Delta_{\mathrm{I}}$ in ultra-thin $(N<6)$ $\mathrm{PbI}_{2} \quad \mathrm{NF}$ embedded into these mediums should be different. In NF of a higher thickness $(N>6)$, where the 
influence of interface phonons is low as compared to that of the confined ones, the total shift should be practically the same for both barrier mediums (curves 3).

Dependences of the energy transitions into the ground exciton state $\left(E_{e x}\right)$ on the NF thickness and temperature are adduced in Figs 2 and 3. The value of this energy is defined by three factors, namely:

1) spatial confinement that causes dimensional quantization of single-particle states in QW, and, as a consequence, shifting their levels to the side of higher energies;

2) self-polarization of the heterojunction planes, which results in shifting the levels in the same direction;

3) interaction between excitons and phonons, which leads to down-shifting its energy.

Being influenced by the first two factors, the bottom of the ground exciton band in $2 \mathrm{H}-\mathrm{PbI}_{2} \mathrm{NF}$ is shifted relatively to its position in bulk crystal to the side of higher energies. In accord with [5], at the temperature $T=77 \mathrm{~K}$ the minimum of the band related with exciton transitions in transmission spectra of $\mathrm{PbI}_{2}$ microcrystals grown in a glass matrix is shifted to the short-wave range as compared to its position in bulk crystal by the value between $35 \mathrm{meV}$ (for $N=7$ ) and $60 \mathrm{meV}$ (for $N=4$ ). The difference between these values is $25 \mathrm{meV}$.
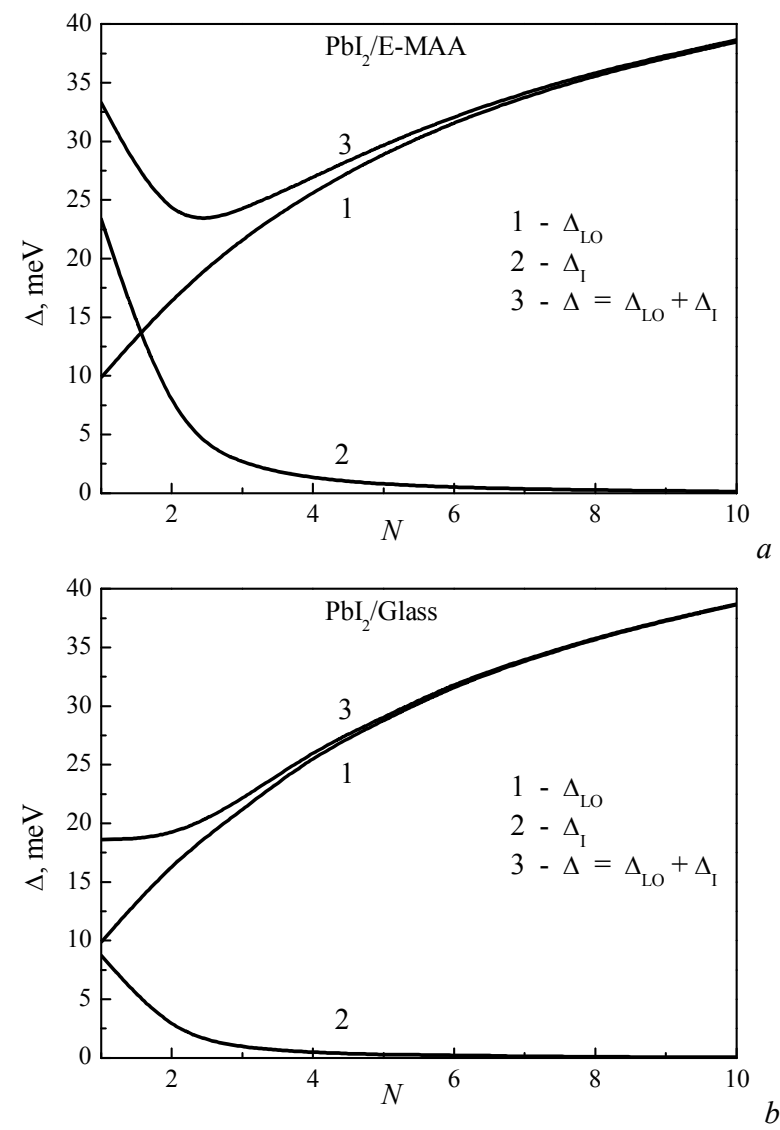

Fig. 1. Value of the long-wave shift $(\Delta)$ inherent to the bottom of the ground exciton energy subband in NP of various thicknesses $(N)$ and its partial components caused by interaction with limited $\left(\Delta_{\mathrm{LO}}\right)$ and interface $\left(\Delta_{\mathrm{I}}\right)$ virtual phonons within the temperature range close to absolute zero.

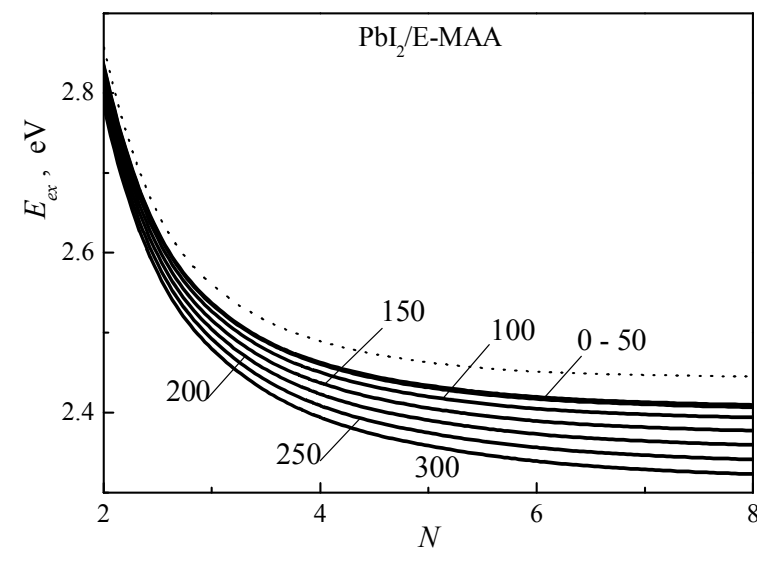

$a$

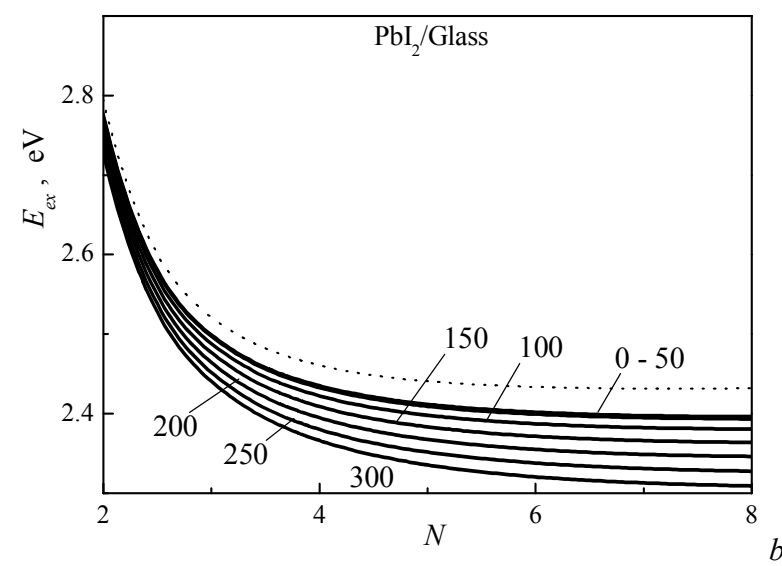

Fig. 2. Dependence of the energy of transition into the ground exciton state on the NP thickness $N$ for various values of the absolute temperature (see numbers near curves).

The calculated by us dependence of the energy corresponding to the exciton transition $E_{e x}$ on the NF thickness with account of SC and SP effects is shown with the dashed line in Fig. 2. For $N \leq 6$, the above short-wave shift of the exciton band bottom exceeds the shift of the opposite direction caused by EPI. For $N \geq 7$, due to the growing influence of LO-phonons, the exciton band bottom shifts to the long-wave range, and the shift is more pronounced, the higher is the NF thickness (solid lines in Fig. 2, numbers near which stand for temperature). Differences between $E_{e x}$ values for the same thickness of $2 \mathrm{H}-\mathrm{PbI}_{2} \mathrm{NF}$ embedded into E-MAA polymer or glass are explained by the difference in dielectric properties of barrier mediums, which define manifestation of the SP effect, and in ultra-thin films of interaction with I-phonons, as well.

In accordance with calculation results adduced in Fig. 2, essential temperature changes in the $E_{e x}$ value begin at $T \geq 75 \mathrm{~K}$. With increasing the temperature of $\mathrm{NF}$ possessing the thickness 4 to 6 layers of $2 \mathrm{H}-\mathrm{PbI}_{2}$ up to the room one, the calculated value of the long-wave shift for the energy of exciton transitions increases approximately by $80 \mathrm{meV}$, which coincides with the data in [5]. 

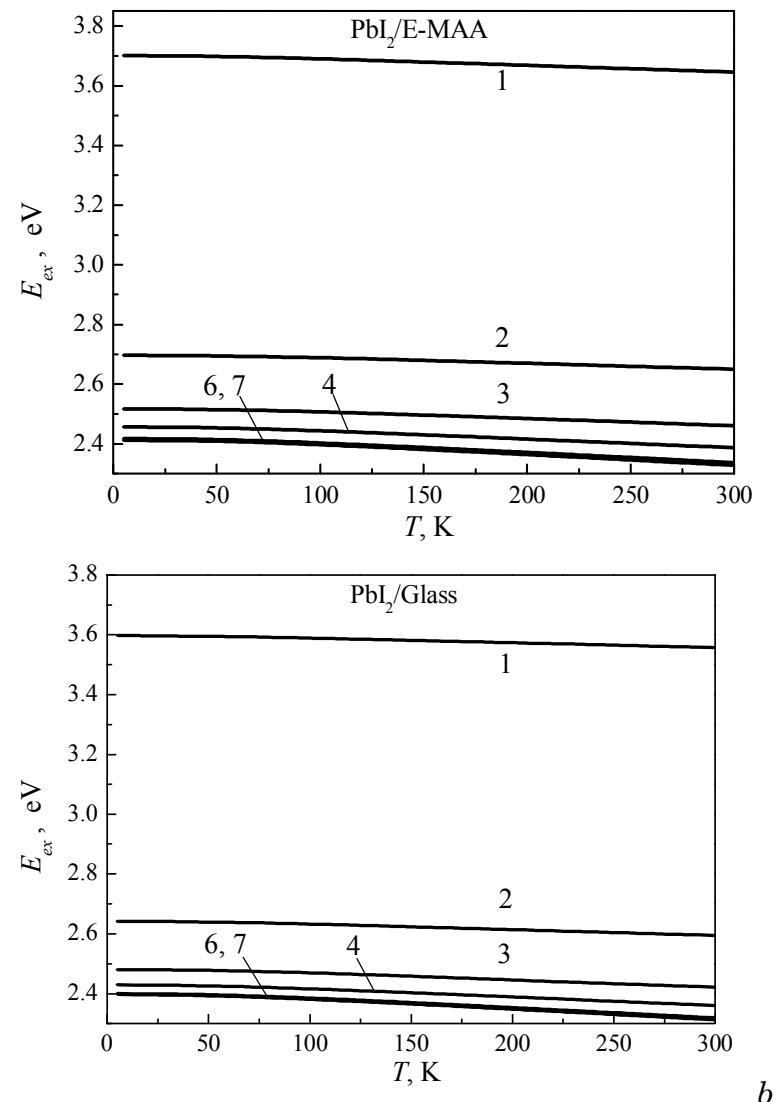

Fig. 3. Temperature changes in the energy of transition into the ground exciton state (NP thickness $N$ is marked with ciphers near the curves).

The difference between energies of exciton transitions in $2 \mathrm{H}-\mathrm{PbI}_{2} \mathrm{NF}$ calculated for $\mathrm{N}=4$ and $\mathrm{N}=6$ is $34 \mathrm{meV}$ in the case of glass matrix at $75 \mathrm{~K}$, which agrees well with the data of transmission spectra measurements [5] $(25 \mathrm{meV}$ at $77 \mathrm{~K})$. The analogous difference calculated for $N=4$ and $N=7$ in $2 H-\mathrm{PbI}_{2} / \mathrm{E}$ MAA at the temperature $5 \mathrm{~K}$ is close to $44 \mathrm{meV}$, which agrees with the data of absorption spectra measurements [6] (50 meV at $2 \mathrm{~K})$.

The temperature dependence for $E_{e x}$ in the range $T>75 \mathrm{~K}$ is practically linear and is enhanced with growth of the NF thickness (Fig. 3). The latter is explained by the growing efficiency of interaction with LO-phonons when increasing the NF thickness.

\section{Conclusions}

Our theoretical investigations performed using the Green function method within the frameworks of effective mass approximation for carriers and the model of dielectric continuum for phonons have shown that the exciton energy spectrum in the $2 \mathrm{H}-\mathrm{PbI}_{2} \mathrm{NF}$ embedded into glass or E-MAA polymer is formed predominantly under action of three factors: spatial confinement of quasi-particles, self-polarization of heterojunction planes and electron-phonon interaction. The influence of the first of them - shift to the side of higher energies - is the most essential for all the considered NF thickness values, and in ultra-thin films it is determinative.

The role of SP and EPI effects is different. As a consequence of the former, the exciton energy is shifted to the side of higher values, while the latter causes its shift in the opposite direction. In thin NF (not exceeding 6 layers of lead di-iodide), contribution of the SP effect to the exciton energy transition value is higher than the EPI one. At the same time, in NF of a higher thickness the influence of the EPI effect is more essential. The value of long-wave shift caused by interaction with phonons grows with the nanosystem temperature. This growth can be essential at temperatures higher than $75 \mathrm{~K}$ as a consequence of growth of filling numbers for phonon states (predominantly of the LO-type). Accordingly, temperature growth up to the room one causes decrease in the exciton transition energy in both considered NF types by 50 to $80 \mathrm{meV}$, in dependence of their thickness.

\section{References}

1. L. Fornaro, E. Saucedo, L. Mussio, A. Goncharov, Growth of lead iodide platelets for room temperature X-ray detection by the vapor transport method // Proc. SPIE 4507, p. 90-98 (2001).

2. R. Ahuja, H. Arwin, A. Ferrera da Silva et al., Electronic and optical properties of lead iodide // J. Appl. Phys. 92(12), p. $7219-7224$ (2002).

3. F.V. Motsnyi, V.G. Dorogan, Z.D. Kovalyuk, S.M. Okulov, Peculiarities of exciton and EPR spectra of $2 \mathrm{H}-\mathrm{PbI}_{2}$ layered crystals with high concentration of Mn impurity // Ukr. J. Phys. 51(5), p. 483-486 (2006).

4. V.B. Kapustyanyk, R.M. Pasternak, A.Z. Kalush et al., Exciton spectra of layered $\mathrm{PbI}_{2}$ and $\mathrm{PbI}_{2}: \mathrm{Zr}$ crystals // J. Appl. Spectr. 74(2), p. 252-257 (2007).

5. A.S. Ablitzova, V.F. Aguekian, A.Yu. Serov, Optical spectra of microcrystals of a layer semiconductor $\mathrm{PbI}_{2}$ in porous glass matrices // Fizika $i$ tekhnika poluprov. 32(2), p. 151-154 (1998), in Russian.

6. A. Yamamoto, H. Nakahara, S. Yano, T. Goto, and A. Kasuya, Exciton dynamics in $\mathrm{PbI}_{2}$ ultra-thin microcrystallites // Phys. stat. sol. (b), 224(1), p. 301-305 (2001).

7. N. Preda, L. Mihut, I. Baltog, T. Velula, $\mathrm{V}$. Teodorescu, Optical properties of lowdimensional $\mathrm{PbI}_{2}$ particles embedded in polymer matrix // J. Optoelectron. \& Adv. Materials, 8(3), p. 909-913 (2006).

8. K. Gauthorn, J-S. Lauret, L. Doyennette et al., Optical spectroscopy of two-dimensional layered $\left(\mathrm{C}_{6} \mathrm{H}_{5} \mathrm{C}_{2} \mathrm{H}_{4}-\mathrm{NH}_{3}\right)_{2}-\mathrm{PbI}_{4}$ perovskite // Opt. Exp. 18(6), p. 5912-5918 (2010). 
9. D.B. Mitzi, K. Chondroulis, and C.R. Kagan, Organic-inorganic electronics // IBM J. Res. Develop. 45, p. 29-45 (2001).

10. I. Saikumar, Shahab Ahmad, J.J. Baumberg, and G. Vijaya Praksh, Fabrication of excitonic luminescent inorganic-organic hybrid nano- and microcrystals // Scripta Materialia, 67, p. 834-837 (2012).

11. I.Kh. Akopyan, O.N. Volkova, B.V. Novikov, B.I. Venzel, Size effects in the optical spectra of the microcrystals $\mathrm{PbI}_{2}$ and $\mathrm{HgI}_{2}$ // Fizika tverdogo tela, 39(3), p. 468-473 (1997), in Russian.

12. R. Zheng, M. Matsuura, T. Taguchi, ExcitonLO-phonon interaction in zinc-compound quantum wells // Phys. Rev. B, 61(15), p. 99609963 (2000).

13. R.T. Senger, K.K. Bajaj, Binding energy of excitons in II-VI compound-semiconductor based quantum well structures // Phys. stat. sol. (b) 241(8), p. 1896-1900 (2004).

14. I.V. Ponomarev, L.I. Deych, V.A. Shuvayev, A.A. Lisyansky, Self-consistent approach for calculations of exciton binding energy in quantum wells // Physica E, 25, p. 539-553 (2005).

15. V.M. Kramar, M.V. Tkach, Exciton-phonon interaction and exciton energy in semiconductor nanofilms // Ukr. J. Phys. 54(10), p. 1027-1035 (2009).

16. Z.D. Kovalyuk, O.A. Politanska, O.N. Sydor, V.T. Maslyuk, Electrical and photoelectric characteristics of structures based on InSe and GaSe layered semiconductors irradiated with 12.5 MeV electrons // Fizika i tekhnika poluprov. 42(11), p. 1321-1326 (2008), in Russian.

17. L. Wendler, R. Pechstedt, Dynamical screening, collective excitations, and electron-phonon interaction in heterostructures and semiconductor quantum wells // Phys. stat. sol. (b), 141(1), p. 129150 (1987).

18. N. Mori, T. Ando, Electron-optical-phonon interaction in single and double heterostructures // Phys. Rev. B, 40(9), p. 6175-6188 (1989).
19. M.V. Tkach, Quasi-particles in Nanoheterosystems. Quantum Dots and Wires: A Manual. Chernivtsy Univ. Press, Chernivtsy, 2003 (in Ukrainian).

20. L.V. Keldysh, Coulomb interaction in thin films of semiconductors and semimetals // Pis'ma JETF, 29(11), p. 716-719 (1979), in Russian.

21. M. Kumagai, T. Takagahara, Excitonic and nonlinear optical properties of dielectric quantumwell-structure // Phys. Rev. B 40(12), p. 12359-81 (1989).

22. M.V. Tkach, V.M. Kramar, Electron-phonon interaction and the mechanisms of electron spectrum re-normalization in a flat nanofilm // $\mathrm{Ukr}$. J. Phys. 53(8), p. 810-818 (2008).

23. O.V. Pugantseva, V.M. Kramar, Self-polarization effect and electron-phonon interaction contributions in forming of electron energy spectrum of $\mathrm{PbI}_{2}$ nanofilm embedded in E-MAA copolymer // J. Nano-Electron. Phys. 4(4), 04021(6 p.) (2012).

24. M.V. Tkach, V.M. Kramar, Thermal genesis of the bottom of main electron's energy band in a flat nanofilm // Ukr. J. Phys. 53(11), p. 1110-1118 (2008).

25. M.S. Brodin, I.V. Blonskii, Excitonic Processes in Layered Crystals, Naukova Dumka, Kiev, 1986 (in Russian).

26. O. Madelung, U. Rossler, H. Shulz, NonTetrahedraly Bonded Elements and Binary Compounds. Springer-Verglag, Berlin, 1998.

27. R. Minder, G. Ottaviani, C. Canali, Charge transport in layer semiconductors // J. Phys. Chem. Sol. 37(2), p. 417-424 (1976).

28. I.V. Blonskii, M.S. Brodin, T.N. Sushkevych, Effect of anisotropy of the crystal structure $\mathrm{PbJ}_{2}$ on its spectral properties // Ukr. J. Phys. 22(11), p. 1907-1910 (1977), in Ukrainian.

29. J.A. Brogan, C.C. Berndt, G.P. Simon, D. Hewitt, Physical and relaxation properties of flame-sprayed ethylene-methacrylic acid copolymer // Polymer Eng. \& Sci. 38(11), p. 1873-1881 (1998). 